\title{
Hard X-ray Emission Associated with White Dwarfs
}

\author{
Ian J. O’Dwyer, You-Hua Chu, Robert A. Gruendl, Martín A. Guerrero, Ronald F. \\ Webbink \\ Department of Astronomy, University of Illinois, 1002 W. Green Street, Urbana, IL 61801; \\ iodwyer@astro.uiuc.edu, chu@astro.uiuc.edu, gruendl@astro.uiuc.edu, mar@astro.uiuc.edu, \\ webbink@astro.uiuc.edu
}

Received __; accepted _ 


\begin{abstract}
Inspired by the hard X-ray emission from WD 2226-210, the central star of the Helix Nebula, we have made a systematic search for similar sources by correlating the white dwarf catalog of McCook \& Sion (1999) and the ROSAT PSPC point source catalog of White, Giommi, \& Angelini (2000). We find 76 white dwarfs coincident with X-ray sources at a high level of confidence. Among these sources, 17 show significant hard X-ray emission at energies $>0.5 \mathrm{keV}$. Twelve of these white dwarfs with hard X-ray emission are in known binary systems, in two of which the accretion of the close companion's material onto the white dwarf produces hard X-ray emission, and in the other ten of which the late-type companions' coronal activity emits hard X-rays. One apparently single white dwarf is projected near an AGN which is responsible for the hard Xray emission. The remaining four white dwarfs and two additional white dwarfs with hard X-ray emission appear single. The lack of near-IR excess from the apparently single white dwarfs suggests that either X-ray observations are more effective than near-IR photometry in diagnosing faint companions or a different emission mechanism is needed. It is intriguing that $50 \%$ of the six apparently single white dwarfs with hard X-ray emission are among the hottest white dwarfs. We have compared X-ray properties of 11 hot white dwarfs with different spectral types, and conclude that stellar pulsation and fast stellar winds are not likely the origin of the hard X-ray emission, but a leakage of the high-energy Wien tail of emission from deep in the stellar atmosphere remains a tantalizing source of hard X-ray emission from hot DO and DQZO white dwarfs. A complete survey using the entire ROSAT PSPC archive is needed to enlarge the sample of white dwarfs with hard X-ray emission. Follow-up near-IR photometric observations are needed to verify the existence of late-type companions and high-resolution
\end{abstract}


deep X-ray observations are needed to verify the positional coincidence and to study the X-ray spectral properties in order to determine the origin and nature of the hard X-ray emission.

Subject headings: white dwarfs - binaries: general - stars: coronae - stars: late-type - X-rays

\section{Introduction}

White dwarfs can be sources of soft $(<0.4 \mathrm{keV})$ X-ray emission if their atmospheres have high temperatures and low opacities: $T_{\text {eff }} \gtrsim 23,000 \mathrm{~K}$ for DA white dwarfs with pure hydrogen atmospheres (Jordan et al. 1994), 23,000 $\mathrm{K} \lesssim T_{\text {eff }} \lesssim 54,000 \mathrm{~K}$ for DA white dwarfs containing significant quantities of heavy elements in their atmospheres (Marsh et al. 1997), and $T_{\text {eff }} \gtrsim 100,000 \mathrm{~K}$ for DO and PG 1159 white dwarfs with helium-rich atmospheres (Motch, Werner, \& Pakull 1993). ROSAT observations of such X-ray sources show soft spectra rising toward ROSAT's low-energy limit at $0.1 \mathrm{keV}$. The DO white dwarf KPD $0005+5106(=$ WD $0005+511)$, with its spectrum peaking at $0.2 \mathrm{keV}$, appears to pose an exception and has been interpreted as having cool $\left(2-3 \times 10^{5} \mathrm{~K}\right)$ coronal emission (Fleming, Werner, \& Barstow 1993). No hard X-ray (>0.5 keV) emission is expected from single white dwarfs.

It is thus puzzling that WD 2226-210², the 103,600 K DAO white dwarf (Méndez et al. 1988; Napiwotzki 1999) in the Helix Nebula, appears to be single (Ciardullo et al. 1999), but has hard X-ray emission. ROSAT observations of WD 2226-210 have detected not only a soft spectral component as expected from the white dwarf's photosphere, but also a hard

\footnotetext{
${ }^{1}$ This white dwarf was cataloged with a sign error in declination as WD $2226+210$ by McCook \& Sion (1999).
} 
spectral component peaking at $0.8 \mathrm{keV}$ (Leahy, Zhang, \& Kwok 1994). Recent Chandra observations have confirmed that the hard X-ray emission is unresolved and coincident with the white dwarf; however, the luminosity and variability of the hard X-ray emission is similar to that of a dMe star (Guerrero et al. 2001). Follow-up spectroscopic observations by Gruendl et al. (2001) detected variability in the $\mathrm{H} \alpha$ line profile of WD 2226-210, suggesting the presence of a companion. It is thus possible that WD 2226-210 has an X-ray-emitting dMe companion which is too faint and close to the white dwarf to be detected at visible wavelengths. Based on the I magnitude of WD 2226-210 reported by Ciardullo et al. (1999) and the $J, H$, and $K$ magnitudes from the 2MASS Survey (see Table 3 below), we estimate that the hypothetical companion must have a spectral type later than M5-6 V.

Hard X-ray emission from white dwarfs may be used to infer the presence of a binary companion. Indeed, Fleming et al. (1996) found 9 DA white dwarfs with hard X-ray emission using the ROSAT All-Sky Survey, and all of these 9 white dwarfs have late-type (F, G, K, and M) companions. This has motivated us to search for other white dwarfs which exhibit hard X-ray emission. We have found that 94 white dwarfs cataloged by McCook \& Sion (1999) appear coincident with ROSAT X-ray point sources in the WGA catalog (White, Giommi, \& Angelini 2000, hereafter WGACAT). To confirm the positional coincidence, we have downloaded the ROSAT data, and compared these X-ray images with optical images of the white dwarfs. We have further extracted the ROSAT spectra to examine whether hard X-ray emission is present. For white dwarfs associated with hard X-ray emission, we have used the literature and near-IR photometry to assess the existence of binary companions, and further investigate the origin of hard X-ray emission from the apparently single white dwarfs. This paper reports the results of our study. The sample of white dwarfs with hard X-ray emission and our method of analysis are described in Section 2, the binarity status of the white dwarfs with hard X-ray emission is reported in Section 3 , and the origin and implications of the hard X-ray emission associated with white dwarfs is 
discussed in Section 4. A summary is given in Section 5.

\section{Search for Hard X-ray Sources Associated with White Dwarfs}

To search for X-ray emission from white dwarfs, we have correlated the white dwarfs from the most recent catalog by McCook \& Sion (1999) with the X-ray sources from the WGACAT, a point source catalog generated from all ROSAT PSPC pointed observations made without the boron filter. For an initial identification of positional coincidence, we require better than $1^{\prime}$ agreement between the cataloged positions of the white dwarf and X-ray point source. This is a conservative criterion because: (1) the on-axis point spread function (PSF) of the ROSAT Position Sensitive Proportional Counter (PSPC) is $\sim 40^{\prime \prime}$ at $1 \mathrm{keV}$ and much worse near $0.1 \mathrm{keV}$ (Snowden et al. 1994; Chu, Kwitter, \& Kaler 1993), (2) the coordinates of the white dwarf might be uncertain by up to 30", and (3) the proper motions of the white dwarfs have not been considered and in some cases may be large enough for the star to move more than $1^{\prime}$ between the epochs when the optical and X-ray observations were made.

We find 94 white dwarfs that appear to be associated with point X-ray sources. To confirm the positional coincidence and to examine further the X-ray spectra of these sources, we have retrieved the event files of their ROSAT PSPC observations from the High Energy Astrophysics Science Archive Research Center (HEASARC) at NASA's Goddard Space Flight Center. The PSPC event files are used to extract broad-band $(0.1-2.4 \mathrm{keV})$ X-ray images. These X-ray images are smoothed with a Gaussian with $\sigma=15^{\prime \prime}$ and the resulting X-ray contours at 10, 20, 50, 70, and $90 \%$ of the peak intensity are overplotted on the broad-band optical images retrieved from the Digitized Sky Survey (DSS). To identify the white dwarfs, we have used the finding charts provided by J. Holberg at http://procyon.lpl.arizona.edu/WD/. 
The results of our detailed comparison of the positions of X-ray sources and white dwarfs are presented in Table 1 . Columns 1-3 give the identifications, common names, and spectral types of the white dwarfs; columns 4-6 list the corresponding X-ray sources in the WGACAT, the ROSAT PSPC observations used for the detection, and the exposure times of the PSPC observations; column 7 describes the positional coincidence between the X-ray source and the white dwarf; and columns 8-10 present the counts detected in the soft $(0.1-0.4 \mathrm{keV})$, medium $(0.4-0.9 \mathrm{keV})$, and hard $(0.9-2.0 \mathrm{keV})$ bands, as reported in the WGACAT.

Eighteen of the 94 coincidences we initially identified could not be confirmed, noted as U1-4 in column 7 of Table 1, because the X-ray sources are not convincingly centered on the white dwarf (U1), the positions of the X-ray sources are compromised by the occultation of the PSPC window support structure or the superposition of a bright background (U2), multiple candidates of optical counterparts are present within the PSPC PSF (U3), or the $\mathrm{X}$-ray sources are too faint to be credible (U4).

One notable example of a U4 mis-identification is WD 1910+047, which was discovered and suggested by Margon, Bolte, \& Anderson (1987) to be associated with an Einstein X-ray source. The weak WGACAT source J1912.5+0452 located within 1' from WD 1910+047 was detected in a $2.8 \mathrm{ks}$ PSPC observation (rp400271n00) at a $44^{\prime}$ off-axis position, but not confirmed in a $20 \mathrm{ks}$ PSPC observation (rp400271a01) with identical pointing. We have examined this shallow PSPC observation and concluded that this source is spurious. Using a deep PSPC observation (rp500058a02, $20.6 \mathrm{ks}$ ) centered at 19' from WD 1910+047, we find an X-ray source near the white dwarf, but it is coincident with a star $\sim 80^{\prime \prime}$ SE of the white dwarf and the X-ray spectrum is typical for coronal emission from a late-type star (see Figure 1). This result explains the conclusion of the model atmosphere analysis by Vennes (1990) that the Einstein X-ray source is too luminous for the white dwarf. 
There remain 76 coincidences that are confirmed with a high degree of confidence. We have further extracted X-ray spectra from the PSPC event files for these X-ray sources coincident with white dwarfs. We find 69 dominated by soft X-ray emission $(<0.5 \mathrm{keV})$, as expected from white dwarfs; however, 10 of these also exhibit hard X-ray emission (0.5-2.4 $\mathrm{keV}$ ). The spectra of the remaining 7 sources have characteristics consistent with coronal emission at temperatures of a few $\times 10^{6} \mathrm{~K}$. These PSPC spectra appear "double-peaked" because of the carbon $\mathrm{K} \alpha$ absorption of the PSPC entrance window at $0.24-0.4 \mathrm{keV}$; the hard component $(>0.5 \mathrm{keV})$ is at least $25 \%$ as strong as the soft component $(<0.5 \mathrm{keV})$.

The 17 white dwarfs associated with hard X-ray emission are listed in Table 2, and their images and X-ray spectra are presented in Figure 2. The left panels display DSS images centered on the white dwarfs and overlaid by X-ray contours to illustrate the positional coincidence of the white dwarf and the X-ray source. The white dwarf position is marked when several stars are projected in its vicinity. The right panels display the PSPC spectra of the X-ray sources. For objects overwhelmed by soft X-ray emission, we also plot the spectra with an expanded Y-scale to show the hard component. The PSPC observations listed in Table 1 have been used to extract these X-ray images and spectral information.

Note that Column 10 of Table 1 shows many more white dwarfs with $3 \sigma$ detections in the $0.9-2.4 \mathrm{keV}$ band. However, all except the above 17 white dwarfs are rejected because their coincidences with X-ray source are not confirmed (U1-4 in Column 7) or the WGACAT source counts are contaminated/confused with background hard X-ray sources. For example, some white dwarfs are observed at such large off-axis angles $\left(>40^{\prime}\right)$ that the PSF contains background sources (e.g., WD 0037+312 and WD 1040+451), and some are blended with adjacent sources (e.g., WD 1821+643, WD 1844-654, and WD 0904+511). Under such circumstances, the source detection algorithm of WGACAT does not effectively exclude contaminating background sources within the PSF, resulting in the apparent 
detection of hard X-ray emission.

\section{Binarity of White Dwarfs with Hard X-ray Emission}

White dwarfs by themselves do not emit hard ( $>0.5 \mathrm{keV})$ X-rays. If a white dwarf accretes material from its surroundings, the gravitational energy released may power hard X-ray emission. In general, we can rule out the accretion of interstellar material because white dwarfs usually are not in a dense interstellar environment. We can also rule out the accretion of the planetary nebula produced by a white dwarf's progenitor, as it either has dissipated already or is expanding away at a speed much greater than the escape velocity. Thus, the most likely source to provide material for accretion onto a white dwarf is a binary companion. Alternatively, if a white dwarf has a binary companion with coronal activity, the hard X-ray emission from the companion would appear to be associated with the white dwarf. We therefore suspect that white dwarfs with hard X-ray emission are in binary systems.

A literature search of the 17 white dwarfs with hard X-ray emission reveals that 12 of them are known binaries. Below we describe these 12 known binary systems in $\S 3.1$, and the 5 apparently single white dwarfs in $§ 3.2$.

\subsection{Known Binaries}

WD 0216-032 (VZ Cet) is a detached companion of Mira, a pulsating, cool giant (M2-7 III). Karovska et al. (1997) measured a separation and position angle $\rho=0$ '.578, $\theta=108.3^{\circ}$ (Ep. 1995.9424). Baize (1980) estimated an orbital period of $400 \mathrm{yr}$ for this system (separation $a=0$ !'85), but this result is extremely uncertain (Mason et al. 2001). The white dwarf flickers (Warner 1972), and IUE observations suggest that it may possess 
an accretion disk (Reimers \& Cassatella 1985). The PSPC spectrum shows X-ray emission peaking at $1 \mathrm{keV}$. This hard X-ray emission probably originates from the accretion, since no single cool giants similar to Mira are known X-ray sources.

WD $0347+171$ (V471 Tau) is in the eclipsing binary system V471 Tau with a period of 0.521 days (Nelson \& Young 1970; Guinan \& Sion 1984). The K2 V companion is known for its coronal activity, and coronal mass ejections have been implied from the observations of transient absorption features in the Si III $\lambda 1206$ resonance line (Bond et al. 2001). Using X-ray and EUV eclipses of V471 Tau, Barstow et al. (1992) have demonstrated that the hard X-ray emission indeed originates from the K2 V star.

WD 0429+176 (HZ 9) is in a spectroscopic binary system with a dM4.5e companion and a period of 0.564 days (Lanning \& Pesch 1981; Guinan \& Sion 1984). Late-type main sequence M stars, particularly the ones with Balmer lines in emission (i.e., dMe stars), have been shown to be X-ray emitters (Rucinski 1984). The dM4.5e companion of WD 0429+176 is most likely responsible for the hard X-ray emission detected.

WD $0736+053$ (Procyon B) is a visual binary companion of Procyon A, an F5 IV-V star, with a period of 40.8 yr (Girard et al. 2000). The coronal activity of Procyon A is responsible for the hard X-ray emission (Lemen et al. 1989). In Figure 2, the wavy line shows the motion (proper and orbital) of the white dwarf between the epochs of DSS and ROS AT PSPC observations.

WD 1213+528 (EG UMa) was observed at a large off-axis angle. The PSPC PSF extends over almost $2^{\prime}$, but the white dwarf appears to be the most likely optical counterpart of the X-ray source. WD $1213+528$ has a dM2e binary companion in a 0.668 day period (Lanning 1982).

WD 1255+258J (HD 112313B) is the central star of the planetary nebula LoTr 5 , and 
has a G5 III companion with a rotational period of 5.9 days (Jasniewicz et al. 1996). The orbital period of this binary is unknown, but probably in excess of one year; the binary is unresolved. The strong Ca II H \& K emission lines and broad variable $\mathrm{H} \alpha$ line of the G5 III companion suggest coronal activity. Thus the G5 III companion is probably responsible for the observed hard X-ray emission.

WD 1314+293 (HZ 43A) has a dM3.5e companion (Napiwotzki et al. 1993), resolved at $\rho=2^{\prime \prime} .2, \theta=263^{\circ}$ (Ep. 1996; Mason et al. 2001). This companion is likely responsible for the hard X-ray emission detected.

WD 1631+781 (1ES 1631+78.1) has an unresolved dM4e companion (Catalan et al. 1995). From the lack of detectable radial velocity variations (Sion et al. 1995), we conclude that the orbital period probably exceeds one year. Fluctuations in $\mathrm{H} \beta$ emission from the dM4e companion mark it as the probable site of hard X-ray emission.

$W D 1633+572$ (GJ 630.1B) is the common proper motion companion $\left(\rho=266^{\prime \prime} 19, \theta=\right.$ $22.46^{\circ}$ at Ep. 2000.0 from an astrometric fit to six DSS plates) of the variable star CM Dra (Greenstein 1986). CM Dra is itself an eclipsing binary containing two dM3-4e stars with a period of 1.26 days (Lacy 1977). The proper motion of CM Dra is so large that it must be considered when comparing the DSS and ROSAT PSPC images. In Figure 2, we have drawn an arrow to show the proper motion of CM Dra between the optical and X-ray epochs. The X-ray source is centered on CM Dra, rather than the white dwarf; therefore, the hard X-ray emission clearly originates from the dM3-4e binary.

WD 1634-573 (HD 149499B) is in a wide binary system $\left(\rho=1^{\prime \prime} .319, \theta=51.71^{\circ}\right.$ at Ep. 1991.25, from Hipparcos) with a K2 Ve companion (Wegner 1979, 1981). The emission lines in the companion's spectrum are indicative of coronal activity, which may be responsible for the hard X-ray emission. 
WD 1944-421 (V3885 Sgr) is a cataclysmic variable with orbital period 0.216 days (Downes et al. 2001). Among our sample of 17 white dwarfs with hard X-rays, this is the only one whose hard X-ray component is stronger than the soft X-ray component, indicating a different emission mechanism. The X-ray emission from WD 1944-421 must originate from the accretion of material from a binary companion onto the surface of the white dwarf (Patterson 1994).

WD 2154-512 (GJ 841B) is a visual companion of GJ 841A $\left(\rho=27^{\prime \prime} 93, \theta=251.5^{\circ}\right.$, Ep. 1987.23, from measurements on 2 DSS plates), which consists of two chromospherically active dM3-5e stars with an orbital period of 1.124 days (Jeffries \& Bromage 1993). The dMe stars in GJ 841A are most likely responsible for the hard X-ray emission detected.

\subsection{The Apparently Single White Dwarfs}

WD 0339-451 has an X-ray spectrum similar to those associated with stellar coronal emission. The temperature of this DA white dwarf is unknown, but its photospheric emission probably does not contribute much to the X-ray emission detected since the spectrum below $0.5 \mathrm{keV}$ does not rise toward $0.1 \mathrm{keV}$ as expected.

WD $1134+300$ (GJ 433.1) is a DA2 white dwarf that has been used as a spectrophotometric standard star (Massey \& Gronwall 1990; Hawarden et al. 2001). Its PSPC spectrum shows a distinct peak at $0.8-0.9 \mathrm{keV}$. Detailed inspection shows that the soft X-ray emission is centered at the white dwarf, but the hard X-ray emission is centered at a position $\sim 41^{\prime \prime}$ northwest of the white dwarf, where an AGN has been identified by Mason et al. (2000). This background AGN must be responsible for the hard X-ray emission.

WD 1159-034 (GW Vir), the prototype of PG 1159 white dwarfs, is a well-known pulsating variable (Winget, Hansen, \& van Horn 1983). The faint hard X-ray emission is 
centered on the white dwarf, but the spectrum is too noisy for detailed spectral analysis.

$W D 1234+481(P G 1234+481)$ does not have X-ray emission peaking at $\sim 0.9 \mathrm{keV}$ as the other white dwarfs associated with hard X-ray emission do. However, the spectrum of WD $1234+481$ appears to show excess emission at $0.4-0.6 \mathrm{keV}$.

$W D 1333+510(P G 1333+510)$ is detected at a large off-axis angle, but it is the closest source, among three, to the peak of the X-ray emission. The X-ray spectrum is similar to those of stellar coronae.

\section{Discussion}

X-ray emission from white dwarfs is expected to be soft, but roughly $20 \%$ of X-ray sources associated with white dwarfs exhibit a hard X-ray component $(>0.5 \mathrm{keV})$. What is the origin of hard X-ray emission associated with white dwarfs? We initially speculated that all white dwarfs with hard X-ray emission possess binary companions, but our literature search reveals that 12 of the 17 white dwarfs with hard X-ray emission are known to be in binary or multiple systems, one is superposed by chance near a background AGN, and the remaining four are apparently single white dwarfs. Below we argue that the binary companions are indeed directly or indirectly responsible for the hard X-ray emission associated with white dwarfs in binary systems, use 2MASS photometry to assess the existence of late-type companions, and discuss the hard X-ray emission from the hottest apparently single white dwarfs. 


\subsection{Hard X-ray Emission Associated with White Dwarfs in Binary Systems}

Two of our 12 white dwarfs in binary systems are known to accrete material from their companions: Mira and the cataclysmic variable V3885 Sgr. The accretion of the companion's material onto the surface of the white dwarf produces hard X-ray emission (Patterson 1994). Of the ten non-accreting systems, six have one or two dMe companions, two have a K2 V companion, one has an F5 IV-V companion, and one has a G5 III companion. These late-type companions either are known for their active coronae or have emission lines indicating coronal activity; therefore, these companions are most likely responsible for the observed hard X-ray emission. In some cases, the origin of the hard X-ray emission has been unambiguously established to be the late-type companions by either positional coincidence (e.g., WD 1633+572) or observations of eclipses (e.g., WD 0347+171).

To confirm that these late-type companions are the source of the hard X-ray emission, we compare the hard X-ray luminosities and plasma temperatures of the binary systems with those expected from single late-type stars. We have fitted thin plasma emission models (Raymond \& Smith 1977) to the 0.5-2.4 keV portion of the spectra for the objects with sufficient counts in this energy band. The resultant plasma temperature $k T$ and X-ray luminosity $L_{\mathrm{X}}$ in the $0.5-2.4 \mathrm{keV}$ band are given in Table 2 . The $L_{\mathrm{X}}$ of white dwarfs with late-type companions are completely consistent with those seen in $\mathrm{dM}$ and $\mathrm{K}$ stars, $10^{27}-10^{29} \operatorname{ergs~s}^{-1}$ (Schmitt, Fleming, \& Giampapa 1995; Marino, Micela, \& Peres 2000). The best-fit plasma temperatures are also in the range for stellar coronae. We further assess whether coronal activities of the late-type companions may be induced by binary interaction by correlating the projected binary separation, $a$, with the X-ray luminosity (see Table 2). No clear correlation is seen. This lack of correlation may be caused by the small number of binary systems in our sample and the wide range of physical parameters involved. The cataclysmic variable WD 1944-421 (V3885 Sgr) has the highest hard X-ray 
luminosity, which is also in accord with the expectation of accretion from a Roche-lobe-filled companion. Thus, the hard X-ray emission from white dwarfs in known binary systems can be explained by the presence of their companions.

\subsection{Apparently Single White Dwarfs}

The remaining four white dwarfs associated with hard X-ray emission appear to be single. If these white dwarfs contain previously-unknown, late-type companions, near-IR photometry may reveal these companions (e.g., Green, Ali, \& Napiwotzki 2000). We have obtained $J H K$ photometric measurements of white dwarfs with hard X-rays available in the second incremental data release of the Two Micron All Sky Survey (2MASS), and listed them in Table 3, along with optical photometry from McCook \& Sion (1999). In the case of WD 1633+572 where the white dwarf and the binary dMe companions are resolved by 2MASS, separate entries are given. To enlarge the sample for comparison, we have added two additional white dwarfs with hard X-ray emission that are not from our survey: WD 0005+511 (= KPD 0005+5106; its hard X-ray emission will be discussed in $\S 4.3)$ and WD 2226-210 (= the central star of the Helix Nebula; its hard X-ray emission is described in $\S 1)$.

Clear near-IR excess is observed in the four known binary systems, but not in the apparently single white dwarfs. The lack of near-IR excess, in conjunction with known distances, places constraints on the possible companions of the white dwarfs. The constraint is more stringent for white dwarfs at smaller distances because it would be harder to hide a companion. The nearest apparently single white dwarf with hard X-ray emission is WD $1134+300$ at 15.3 pc. We estimate that its near-IR magnitudes can hide only a brown dwarf companion more than 3 mag fainter than an M7 V star. Therefore, WD 1134+300 has no stellar-mass companions, and the AGN projected within the PSPC PSF (Mason et 
al. 2000) is solely responsible for the hard X-ray emission observed.

Two other white dwarfs with hard X-ray emission have known distances, WD 1234+481 and WD 2226-210. Their lack of near-IR excess indicates that WD 1234+481 can hide a companion of spectral type M7 V or later, and WD 2226-210 later than M5-6 V. If these two apparently single white dwarfs indeed have faint, late-type companions, then the hard X-ray emission detected by $R O S A T$ is more effective than the near-IR excess detected by 2MASS in diagnosing the existence of a faint late-type companion.

\subsection{Hard X-ray Emission from the Hottest Apparently Single White Dwarfs}

Two of the apparently single white dwarfs associated with hard X-rays from our survey are among the hottest known: WD 1159-034 and WD 2226-210. To compare these two hot white dwarfs to the one that has been suggested to possess a corona, KPD 0005+5106, we have retrieved an archival ROSAT PSPC pointed observation (rf200428n00) that was made with the boron filter for an exposure time of $5 \mathrm{ks}$. As shown in Figure 3, the PSPC spectrum of KPD 0005+5106 shows not only the soft atmospheric emission below $0.5 \mathrm{keV}$, but also hard X-ray emission near $1 \mathrm{keV}$. This hard X-ray emission was not detected previously by Fleming, Werner, \& Barstow (1993) using the ROSAT All-Sky Survey observation of exposure time $504 \mathrm{~s}$. As the $5 \mathrm{ks}$ pointed observation detected only $24 \pm 6$ counts in the $0.5-2.4 \mathrm{keV}$ band, if the hard X-ray flux is constant, we expect only

$2.4 \pm 1.5$ hard X-ray counts in a $504 \mathrm{~s}$ exposure. The non-detection of the hard component by Fleming, Werner, \& Barstow (1993) thus does not imply a temporal variation. The presence of hard X-ray emission from KPD 0005+5106 requires a plasma temperature at least $10^{6} \mathrm{~K}$, much higher than that suggested by Fleming, Werner, \& Barstow (1993), and may be responsible for photoionizing O VIII and producing the recombination lines observed (Werner \& Heber 1992; Sion \& Downes 1992). We have examined the 2MASS 
$J H K$ photometric data for KPD 0005+5106 (see Table 3), and find no evidence for a near-IR excess. Thus, KPD $0005+5106$ is another apparently single hot white dwarf with hard X-ray emission.

While our statistical sample is extremely limited, it is intriguing that $\sim 50 \%$ of the apparently single white dwarfs with hard X-ray emission are among the hottest known white dwarfs: 120,000 K for WD 0005+511 (=KPD 0005+5106; Werner, Heber, \& Fleming 1994), 140,000 K for WD 1159-034 (= PG 1159-034; Dreizler \& Heber 1998), and 103,600 K for WD 2226-210 (= CSPN of the Helix; Napiwotzki 1999). Is it possible that the hard X-ray emission is the high-energy Wien tail of the blackbody emission from deep in the stellar atmosphere? It is beyond the scope of this paper to model the atmospheres of these white dwarfs and answer this question theoretically. Instead, we will examine X-ray observations of the hottest white dwarfs to search for trends in their hard X-ray properties in order to gain insight into the origin of their hard X-ray emission.

We first examine X-ray properties of PG 1159 stars. Many pointed ROSAT PSPC observations of PG 1159 stars were made with the boron filter. These X-ray sources will be absent in the WGACAT, and it is necessary to search the ROSAT archive for pointed and serendipitous observations of PG 1159 stars. PG 1159 stars are divided into two groups: with and without planetary nebulae (PNs). ROSAT observations of cataloged PNs have been analyzed and reported by Guerrero, Chu, \& Gruendl (2000); PSPC observations of five PNs with PG 1159 central stars are available. Of these five, WD 0726+133 (in Abell 21; PG 1159) and WD 2333+301 (in Jn 1; DOZ.3) are not detected; WD 2117+342J (in MWP 1; DO) is detected at a 40' off-axis position in the PSPC field-of-view (this paper), but the poor PSF does not allow us to assess accurately whether faint hard X-ray emission exists; WD 0044-121 (in NGC 246; PG 1159) and WD 1821+643 (in K 1-16; DOZ.4) are centered in pointed PSPC observations, but only soft $(<0.5 \mathrm{keV})$ X-ray emission from the 
white dwarf is detected. Four PG 1159 stars without PNs have pointed PSPC observations: WD 0122-753J (= RX J0122-7521; DO), WD 1144+004 (= PG 1144+005; DQZO1), WD 1159-034 (= PG 1159-034; DQZO.4), and WD 1501+664 (= PG 1501+661; DZ1); all four have been included in our survey listed in Table 1. Soft X-ray emission is detected in these four PG 1159 stars without PNs, but 3- $\sigma$ detection of hard X-rays is obtained only for PG 1159 itself.

We have further combed Table 1 for hot white dwarfs similar to WD $0005+511$ or WD 2226-210, and find a hot DO white dwarf, WD 1522+662, and a hot DAO white dwarf, WD $1957+225$ at the center of the Dumbbell Nebula. These four hot DO and DAO white dwarfs and the above nine PG 1159 white dwarfs will be discuss below in more detail. The spectral type, stellar effective temperature, visual magnitude, and ROSAT observations of these 13 hot white dwarfs are summarized in Table 4. To illustrate the spectral properties of the 11 hot white dwarfs whose X-ray emission has been detected by ROSAT PSPC observations, in Figure 4 we present their soft X-ray images in the $0.1-0.4 \mathrm{keV}$ band and the hard X-ray images in the $0.6-2.4 \mathrm{keV}$ band. It is evident that hard X-ray emission is clearly detected only from WD 0005+511, WD 1159-034, and WD 2226-210, as we have concluded earlier, and possibly detected at a $2 \sigma$ level from WD 0122-753J. The spectral types of these four white dwarfs are DO, DQZO.4, DAO, and DO, respectively.

Below we divide the 11 hot white dwarfs detected in X-rays according to their spectral types, discuss the implications of their hard X-ray properties, and speculate on the possibility of a photospheric origin of the hard X-ray emission.

- DAO (WD 1957+225 and WD 2226-210):

Hard X-ray emission is detected from WD 2226-210 in the Helix Nebula, but not from WD 1957+225 in the Dumbbell Nebula. As these two DAO white dwarfs have similar effective temperatures, and as WD $1957+225$ is only 0.8 mag fainter but has a 
four times longer exposure time, the lack of hard X-ray emission from WD 1957+225 signifies a real difference from WD 2226-210. Furthermore, WD 2226-210 shows temporal variations of its hard X-ray emission and $\mathrm{H} \alpha$ line profile, suggesting the existence of a late dMe companion (Guerrero et al. 2001; Gruendl et al. 2001). Therefore, there is no evidence indicating that deep photospheric emission is the origin of hard X-rays associated with DAO white dwarfs.

- $D O(W D 0005+511, W D 0122-753 J, W D 1522+662$, and WD 2117+342J):

Among these four DO white dwarfs, WD 0005+511 has an unambiguous detection of hard X-rays, and WD 0122-753J has a possible detection of hard X-rays. WD 1522+662 does not show hard X-ray emission, but the ROSAT observation has only $4.7 \mathrm{ks}$ exposure time and WD $1522+662$ is faint with $B=16.4$ mag. WD $2117+342 \mathrm{~J}$ is observed at a $40^{\prime}$ from the center of the PSPC field-of-view and the poor PSF prohibits a conclusive assessment of the existence of faint hard X-ray emission. It may be possible that faint hard X-ray emission can emerge from the hot, deep layer of the photospheres of DO white dwarfs, but more detections are needed to confirm it.

- $D Q Z O(W D 1144+044, W D 1159-034$, and WD 1821+643): WD 1159-034 has hard X-ray emission. WD 1144+044 has a non-detection, but it is slightly fainter than WD 1159-034 and its exposure time is only half as long. WD $1821+643$ in the PN K1-16 is projected near a bright hard X-ray source, a cluster of galaxies surrounding the QSO E1821+643 (Saxton et al. 1997), so it is difficult to determine its hard X-ray properties accurately from Figure 4. We have examined an archival Chandra HETG observation of QSO E1821+643 (PI: C. R. Canizares; $101 \mathrm{ks}$ ); in this observation WD 1821+643 is detected and clearly resolved from QSO E1821+643, but no hard X-ray emission from WD $1821+643$ is seen. 
WD 1159-034 and WD 1821+643 are well-known pulsators (Ciardullo \& Bond 1996), while WD $1144+044$ is not. It is thus unlikely that the hard X-ray emission is related to the stellar pulsation.

\section{- $P G 1159$ and DZ1 (WD 0044-121 and WD 1501+664):}

WD 0044-121 is a PG 1159 star in the PN NGC 246; WD 1501+664 is classified as DZ1 and is the hottest white dwarf (Werner \& Wolff 1999). Neither of these two hot white dwarfs show hard X-ray emission; the high opacity of the H- and He-free atmosphere of WD 1501+664 may be the culprit of its non-detection (Nousek et al. 1986; Werner 1991).

The above sample of hot white dwarfs is very limited, but allows us to eliminate improbable origins of hard X-ray emission. For example, stellar pulsation is not likely to produce hard X-ray emission, as WD 1159-034 is the only pulsator with hard X-ray emission. Stellar winds are not likely to be the origin of hard X-ray emission either, as WD 1159-034 has hard X-rays but no measurable past or ongoing mass loss (Fritz, Leckenby, \& Sion 1990), while appreciable mass loss but no hard X-ray emission has been detected from WD 0044-121 and WD 1821+643 (Koesterke \& Werner 1998). There leaves the tantalizing suggestion that hard X-rays may be emitted by hot DO and DQZO white dwarfs. While it is necessary to model their atmospheres to understand theoretically whether hard X-rays from beneath the atmosphere may leak through, it is also necessary to obtain better high-resolution X-ray images to confirm that the hard X-ray emission is indeed associated with the white dwarfs (as opposed to background objects projected in their vicinity) and high-quality X-ray spectra for detailed spectral analysis. 


\section{Summary}

We have correlated the recent white dwarf catalog by McCook \& Sion (1999) with the ROSAT PSPC point source catalog (WGACAT), and found 76 white dwarfs coincident with X-ray sources at a high level of confidence. We have further found that 17 of these sources show hard X-ray emission $(>0.5 \mathrm{keV})$. Two of these white dwarfs with hard X-ray emission accrete material from their companions, while the other ten have late-type companions that are known to have active coronae and emit hard X-rays. One white dwarf has an AGN projected within the PSPC PSF contributing to the hard X-ray emission. The remaining four white dwarfs (WD 0339-451, WD 1159-034, WD 1234+481, and WD 1333+510) and two additional white dwarfs (WD 0005+511 and WD 2226-210) with hard X-ray emission appear single. The lack of near-IR excess for WD 1234+481 and WD 2226-210 at known distances constrains the possible spectral types of the hidden companions to later than M7 V and M5-6 V, respectively. We suggest that hard X-ray emission may be more effective than near-IR photometry in diagnosing faint, late-type companions of white dwarfs, if the hard X-ray emission associated with the six apparently single white dwarfs indeed originates from hidden companions.

It is intriguing that three of the six apparently single white dwarfs with hard X-ray emission have high stellar effective temperatures. We have searched the ROSAT archive for observations of PG 1159 stars and examined the X-ray properties of a sample of 13 hot white dwarfs with different spectral types. Comparisons among these hot white dwarfs lead to the following conclusions: (1) DAO WD 2226-210 most likely possesses a late dMe companion which emits hard X-rays, (2) stellar pulsation cannot be connected to the hard X-ray emission, (3) fast stellar winds are not likely to be the origin of hard X-ray emission, and (4) the high-energy Wien tail of emission deep in the atmospheres of hot DO and DQZO white dwarfs remains a tantalizing explanation for the hard X-ray emission observed. 
Our statistics are limited by the incompleteness of the WGACAT, which has been derived solely from pointed ROSAT PSPC observations made without filters, while many PSPC observations of white dwarfs were made with a boron filter. A complete survey for white dwarfs with hard X-ray emission using the entire ROSAT archive is needed to enlarge the sample. Follow-up high-resolution deep X-ray observations with Chandra or $X M M-N e w t o n$ are needed to confirm the positional coincidence of the white dwarfs and the X-ray sources, and to study the spectral properties in order to investigate the origin and nature of hard X-ray emission associated with white dwarfs.

We thank the anonymous referee for making critical comments which helped improve our paper. We also thank J. Liebert, R. Napiwotzki, R. Petre, and K. Werner for reading the manuscript and making useful suggestions. This research has made use of the SIMBAD database, operated at CDS, Strasbourg, France, and the Digital Sky Survey produced at the Space Telescope Science Institute under U.S. Government grant NAG W-2166. We have also used data product from the 2MASS, which is a joint project of the University Massachusetts and the Infrared Processing and Analysis Center/California Institute of Technology, funded by NASA and NSF. 


\section{REFERENCES}

Baize, P. 1980, A\&AS, 39, 86

Barstow, M. A., et al. 1992, MNRAS, 255, 369

Bond, H. E., Mullan, D. J., O'Brien, M. S., \& Sion, E. M. 2001, ApJ, 560, 919

Catalan, M. S., Sarna, M. J., Jomaron, C. M., \& Connon Smith, R. 1995, MNRAS, 275, 153

Catchpole, R. W., Robertson, B. S. C., Lloyd Evans, T. H. H., Feast, M. W., Glass, I. S., \& Carter, B. S. 1979, SAAO Circ., 1, 61

Chu, Y.-H., Kwitter, K. B., \& Kaler, J. B. 1993, AJ, 106, 650

Ciardullo, R., \& Bond, H. E. 1996, AJ, 111, 2332

Ciardullo, R., Bond, H. E., Sipior, M. S., Fullton, L. K., Zhang, C.-Y., \& Schaefer, K. G. 1999, AJ, 118, 488.

Dahn, C. C., et al. 1982, AJ, 87, 419

Downes, R. A., Webbink, R. F., Shara, M. M., Ritter, H., Kolb, U., \& Duerbeck, H. W. 2001, PASP, 113, 764

Dreizler, S. \& Heber, U. 1998, A\&A, 334, 618

Fleming, T. A., Snowden, S. L., Pfeffermann, E., Briel, U., \& Greiner, J. 1996, A\&A, 316, 147

Fleming, T. A., Werner, K., \& Barstow, M. A. 1993, ApJ, 416, L79

Fritz, M. L., Leckenby, H. J., \& Sion, E. M. 1990, AJ, 99, 908 
Girard, T. M. et al. 2000, AJ, 119, 2428

Green, P. J., Ali, B., \& Napiwotzki, R. 2000, ApJ, 540, 992

Greenstein, J. L. 1986, AJ, 92, 859

Gruendl, R. A., Chu, Y.-H., O'Dwyer, I. J., \& Guerrero, M. A. 2001, AJ, 122, 308

Guerrero, M. A., Chu, Y.-H., \& Gruendl, R. A. 2000, ApJS, 129, 295

Guerrero, M. A., Chu, Y.-H., Gruendl, R. A., Williams, R. M., \& Kaler, J. B. 2001, ApJ, 553, L55 [Erratum: ApJ, 554, L235]

Guinan, E. F., \& Sion, E. M. 1984, AJ, 89, 1252

Harrington, R. S. \& Dahn, C. C. 1980, AJ, 85, 454

Harris, H. C., Dahn, C. C., Monet, D. G., \& Pier, J. R. 1997, in Planetary Nebulae, IAU Symp. No. 180, ed. H. J. Habing \& H. J. G. L. M. Lamers (Dordrecht: Kluwer), p. 40

Hawarden, T. G., Leggett, S. K., Letawsky, M. B., Ballantyne, D. R., \& Casali, M. M. 2001, MNRAS, 325, 563

Hutchings, J. B., Crampton, D., Cowley, A. P., Schmidtke, P. C., McGrath, T. K., \& Chu, Y.-H. 1995, PASP, 107, 931

Jasniewicz, G., Thevenin, F., Monier, R., \& Skiff, B. A. 1996, A\&A, 307, 200

Jeffries, R. D., \& Bromage, G. E. 1993, MNRAS, 260, 132

Jordan, S., Wolff, B., Koester, D., \& Napiwotzki, R. 1994, A\&A, 290, 834

Karovska, M., Hack, W., Raymond, J., \& Guinan, E. 1997, ApJ, 482, L175 
Koesterke, L., Dreizler, S., \& Rauch, T. 1998, A\&A, 330, 1041

Koesterke, L., \& Werner, K. 1998, ApJ, 500, L55

Lacy, C. H. 1977, ApJ, 218, 444

Lanning, H. H. 1982, ApJ, 253, 752

Lanning, H. H., \& Pesch, P. 1981, ApJ, 244, 280

Leahy, D. A., Zhang, C. Y., \& Kwok, S. 1994, ApJ, 422, 205

Lemen, J. R., Mewe, R., Schrijver, C. J., \& Fludra, A. 1989, ApJ, 341, 474

Margon, B., Bolte, M., \& Anderson, S. F. 1987, AJ, 93, 1229

Marino, A., Micela, G., \& Peres, G. 2000, A\&A, 353, 177

Marsh, M. C., et al. 1997, MNRAS, 287, 705

Mason, K. O. et al. 2000, MNRAS, 311, 456

Mason, B. D., Wycoff, G. L., Hartkopf, W. I., Douglas, G. G., \& Worley, C. E. 2001, The Washington Visual Double Star Catalog, 2001.0 (Washington, DC: US Naval Observatory)

Massey, P., \& Gronwall, C. 1990, ApJ, 358, 344

McCook, G. P. \& Sion, E. M. 1999, ApJS, 121, 1

Méndez, R. H., Kudritzki, R. P., Herrero, A., Husfeld, D., \& Groth, H. G. 1988, A\&A, 190, 113

Motch, C., Werner, K., \& Pakull, M. W. 1993, A\&A, 268, 561

Napiwotzki, R. 1999, A\&A, 350, 101 
Napiwotzki, R., Barstow, M. A., Fleming, T., Holweger, H., Jordan, S., \& Werner, K. 1993, A\&A, 278, 478

Nelson, B., \& Young, A. 1970, PASP, 82, 699

Nousek, J. A., et al. 1986, ApJ, 309, 230

O’Brien, M. S., Bond, H. E., \& Sion, E. M. 2001, ApJ, 563, 971

Patterson, J. 1994, PASP, 106, 209

Perryman, M. A. C. et al. 1997, A\&A, 323, L49

Perryman, M. A. C. et al. 1998, A\&A, 331, 81

Raymond, J. C., \& Smith, B. W. 1977, ApJS, 35, 419

Reimers, D., \& Cassatella, A. 1985, ApJ, 297, 275

Ritter, H., \& Kolb, U. 1998, A\&AS, 129, 83

Rucinski, S. M. 1984, A\&A, 132, L9

Saxton, R. D., Barstow, M. A., Turner, M. J. L., Williams, O. R., Stewart, G. C., \& Kii, T. 1997, MNRAS, 289, 196

Schmitt, J. H. M. M., Fleming, T. A., \& Giampapa, M. S. 1995, ApJ, 450, 392

Sion, E. M., \& Downes, R. A. 1992, ApJ, 396, L79

Sion. E. M., Holberg, J. B., Barstow, M. A., \& Kidder, K. M. 1995, PASP, 107, 232

Snowden, S. L., McCammon, D., Burrows, D. N., \& Mendenhall, J. A. 1994, ApJ, 424, 714

van Altena, W. F. 1969, AJ, 74, 2 
Vennes, S. 1990, ApJ, 361, L65

Warner, B. 1972, MNRAS, 159, 95

Wegner, G. 1979, MNRAS, 187, 17

Wegner, G. 1981, AJ, 86, 264

Werner, K. 1991, A\&A, 251, 147

Werner K., et al. 1997, in Reviews in Modern Astronomy 10, ed. R. E. Schielicke, Astronomische Gesellschaft, 219

Werner, K., \& Heber, U. 1992, in Atmospheres of Early Type Stars, ed. U. Heber \& C. S. Jefferey (Berlin: Springer), 291

Werner, K., Heber, U., \& Fleming, T. 1994, A\&A, 284, 907

Werner, K., \& Wolff, B . 1999, A\&A, 347, L9

White, N., Giommi, P., \& Angelini, L. 2000, The WGA Catalogue of ROSAT Point Sources (WGACAT), available at http://wgacat.gsfc.nasa.gov/wgacat/wgacat.html

Winget, D. E., Hansen, C. J., \& van Horn, H. M. 1983, Nature, 303, 781 


\section{Figure Captions}

Fig. 1.- WD 1910+047. Left: Broad-band Digitized Sky Survey image overlaid by X-ray contours at 10, 20,50,70, and $90 \%$ of the peak value of the X-ray source. The white dwarf is marked by two short lines near the center of the field of view. The X-ray source is clearly associated with a star 80" SE of the white dwarf. Right: ROSAT PSPC spectrum of the X-ray source. The X-ray contours and spectrum are extracted from the 20.6 ks observation rp500058a02.

Fig. 2.- Seventeen white dwarfs associated with hard X-ray emission. Left panels: Broadband Digitized Sky Survey image overlaid by X-ray contours at 10, 20, 50, 70, and $90 \%$ of the peak value of the X-ray source. In some cases, the background is so high that the lower X-ray contours are absent. The white dwarf is at the center of each field of view. When multiple stars are projected in the vicinity of a white dwarf, we mark the white dwarf with two short lines. For white dwarfs with large proper motions, lines are drawn to show the proper motion from the optical epoch to the X-ray epoch. For WD 0736+053, the wavy line shows the proper and orbital motions of the white dwarf; for WD 1633+572, the arrow shows the motion of its common proper motion companion CM Dra. Right panels: ROSAT PSPC spectrum of the X-ray source. In cases where the spectrum is overwhelmed by soft X-ray emission, to show the hard X-ray emission, the spectrum is also plotted with open triangles after being multiplied by a constant factor indicated in the panel. The X-ray contours and spectra are extracted from ROSAT PSPC observations listed in Table 1. 
Fig. 3.- KPD 0005+5106 (=WD 0005+511). Left: Broad-band Digitized Sky Survey image overlaid by X-ray contours at 10,20,50,70, and $90 \%$ of the peak value of the X-ray source. The white dwarf is marked by two short lines near the center of the field of view. Two X-ray sources associated with two different stars are detected. Right: ROSAT PSPC spectrum of the X-ray source associated with the white dwarf KPD 0005+5106. This spectrum was carefully extracted to exclude the X-ray emission from the neighboring source. The X-ray contours and spectrum are extracted from the $5 \mathrm{ks}$ observation rf200428n00 made with a boron filter. Hard X-ray emission at $1 \mathrm{keV}$ is clearly detected.

Fig. 4.- Soft and hard X-ray images of eleven apparently single hot white dwarfs. The soft X-ray images (left panels) are extracted from PSPC observations in the 0.1-0.4 keV energy band, and the hard X-ray images (right panels) in the 0.6-2.4 keV energy band. 
Table 1. White Dwarfs Coincident with WGACAT X-ray Sources

\begin{tabular}{|c|c|c|c|c|c|c|c|c|c|c|}
\hline (1) & $(2)$ & $(3)$ & (4) & $(5)$ & $(6)$ & $(7)$ & (8) & $(9)$ & \multicolumn{2}{|l|}{$(10)$} \\
\hline & & & & ROSAT & Exp. & & X-ray Counts & Reported in & WGACAT & \\
\hline WD & Common & WD & WGACAT & PSPC & Time & Pos. & $0.1-0.4 \mathrm{kev}$ & $0.4-0.9 \mathrm{kev}$ & $0.9-2.4 \mathrm{kev}$ & \\
\hline Number & Name & Type & Number & Obs. \# & {$[\mathrm{ks}]$} & Coin. & {$[\mathrm{cts}]$} & [cts $]$ & [cts] & \\
\hline $0027-636$ & $\ldots$ & DA1 & J0029.9-6324 & 400160 & 2.7 & good & $4081 \pm 64$ & $27 \pm 5$ & $0 \pm 0$ & \\
\hline $0037+312$ & GD 8 & DA1 & J0039.8+3132 & 201045 & 28.4 & good & $2248 \pm 47$ & $106 \pm 10$ & $65 \pm 8$ & \\
\hline $0048-294$ & FOCAP SGP2:31 & DA & J0051.2-2910 & 700275 & 24.5 & $\mathrm{U} 1,2$ & $46 \pm 7$ & $11 \pm 3$ & $24 \pm 5$ & \\
\hline $0050-332$ & GD 659 & DA1 & J0053.2-3300 & 200410 & 3.4 & good & $3787 \pm 62$ & $27 \pm 5$ & $3 \pm 2$ & \\
\hline $0116-231$ & GD 695 & DA3 & J0118.6-2254 & 100376 & 17.0 & good & $50 \pm 7$ & $5 \pm 2$ & $1 \pm 1$ & \\
\hline 0122-753J & & $\mathrm{DO}$ & J0122.8-7521 & 300369 & 5.6 & good & $3130 \pm 56$ & $98 \pm 10$ & $8 \pm 3$ & \\
\hline $0131-163$ & GD 984 & $\mathrm{DA} 1+\mathrm{dM}$ & J0134.4-1607 & 200485 & 0.9 & good & $633 \pm 25$ & $4 \pm 2$ & $0 \pm 0$ & 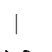 \\
\hline $0136+251$ & PG 0136+251 & DAp1 & J0138.8+2523 & 200539 & 2.1 & good & $550 \pm 24$ & $5 \pm 2$ & $0 \pm 0$ हृ & త్ \\
\hline 0216-032 & VZ Cet & $\mathrm{DA}+\mathrm{M} 2-7 \mathrm{III}$ & J0219.3-0258 & 201501 & 9.1 & good & $5 \pm 2$ & $18 \pm 4$ & $40 \pm 6$ & \\
\hline $0304+154$ & $\ldots$ & $\mathrm{DC}$ & J0307.0+1540 & 800104 & 7.7 & $\mathrm{U} 1,2$ & $48 \pm 7$ & $11 \pm 3$ & $5 \pm 2$ & \\
\hline $0320-539$ & LB 1663 & DA1.5 & J0322.2-5345 & 800307 & 21.5 & good & $2534 \pm 50$ & $14 \pm 3$ & $1 \pm 1$ & \\
\hline $0333-350$ & $\ldots$ & $\mathrm{DA}$ & J0335.5-3449 & 600127 & 18.0 & good & $1480 \pm 38$ & $20 \pm 4$ & $5 \pm 2$ & \\
\hline $0339-451$ & $\ldots$ & $\mathrm{DA}$ & J0341.4-4500 & 900495 & 48.6 & good & $64 \pm 8$ & $22 \pm 5$ & $18 \pm 4$ & \\
\hline $0347+171$ & V471 Tau & $\mathrm{DA} 2+\mathrm{K} 2 \mathrm{~V}$ & J0350.4+1714 & 200107 & 31.8 & good & $6538 \pm 81$ & $688 \pm 26$ & $809 \pm 28$ & \\
\hline $0416-550$ & $\ldots$ & $\mathrm{DA}$ & J0417.1-5457 & 600456 & 17.8 & good & $254 \pm 16$ & $16 \pm 4$ & $11 \pm 3$ & \\
\hline $0425+168$ & EGGR 37 & DA2 & J0428.6+1658 & 200083 & 2.9 & good & $33 \pm 6$ & $3 \pm 2$ & $0 \pm 0$ & \\
\hline $0426+588$ & GJ 169.1B & $\mathrm{DC}+\mathrm{M} 4$ & $\mathrm{~J} 0431.1+5859$ & 201114 & 3.5 & good & $22 \pm 5$ & $4 \pm 2$ & $4 \pm 2$ & \\
\hline $0429+176$ & HZ 9 & $\mathrm{DA} 2+\mathrm{dM} 4.5 \mathrm{e}$ & J0432.4+1744 & 200443 & 20.3 & good & $384 \pm 20$ & $180 \pm 13$ & $161 \pm 13$ & \\
\hline 0443-037J & RE J0443-034 & DA & J0443.0-0346 & 200997 & 10.1 & good & $14710 \pm 121$ & $336 \pm 18$ & $42 \pm 6$ & \\
\hline $0446-789$ & $\ldots$ & DA3 & J0443.6-7851 & 201073 & 8.3 & good & $74 \pm 9$ & $1 \pm 1$ & $1 \pm 1$ & \\
\hline $0518-105$ & RE J0521-102 & DA2 & J0521.3-1029 & 200830 & 5.3 & good & $370 \pm 19$ & $1 \pm 1$ & $0 \pm 0$ & \\
\hline $0531-022$ & RE J0534-021 & DA2 & J0534.3-0213 & 200932 & 8.0 & good & $284 \pm 17$ & $17 \pm 4$ & $6 \pm 2$ & \\
\hline $0548+000$ & GD 257 & DA1 & J0550.6+0005 & 200585 & 4.4 & good & $1404 \pm 38$ & $18 \pm 4$ & $0 \pm 0$ & \\
\hline $0558-756$ & $\ldots$ & DO & J0556.9-7540 & 201245 & 18.5 & $\mathrm{U} 2,3$ & $10 \pm 3$ & $5 \pm 2$ & $13 \pm 4$ & \\
\hline
\end{tabular}


Table 1-Continued

\begin{tabular}{|c|c|c|c|c|c|c|c|c|c|}
\hline$(1)$ & $(2)$ & $(3)$ & (4) & $\begin{array}{c}(5) \\
\text { BOSAT }\end{array}$ & $\begin{array}{r}(6) \\
F x n\end{array}$ & $(7)$ & $\stackrel{(8)}{\text { X-rav Count }}$ & $\begin{array}{c}(9) \\
\text { Beported in }\end{array}$ & $\begin{array}{c}(10) \\
\text { WGACAT }\end{array}$ \\
\hline WD & Common & WD & WGACAT & PSPC & Time & Pos. & $0.1-0.4 \mathrm{kev}$ & $0.4-0.9 \mathrm{kev}$ & $0.9-2.4 \mathrm{kev}$ \\
\hline Number & Name & Туре & Number & Obs. \# & {$[\mathrm{ks}]$} & Coin. & [cts] & [cts] & [cts] \\
\hline $0651-020$ & GD 80 & DA1 & J0654.2-0209 & 201363 & 0.4 & good & $70 \pm 8$ & $1 \pm 1$ & $0 \pm 0$ \\
\hline 0715-704J & RE J0715-705 & DA1 & J0715.2-7025 & 400321 & 2.8 & good & $5439 \pm 74$ & $27 \pm 5$ & $0 \pm 0$ \\
\hline $0718-316$ & IN CMa & $\mathrm{DAO}+\mathrm{dM}$ & J0720.7-3146 & 300338 & 3.2 & good & $411 \pm 20$ & $4 \pm 2$ & $2 \pm 1$ \\
\hline $0734-143$ & $\ldots$ & $\mathrm{DA}$ & J0736.6-1428 & 500295 & 9.1 & U3 & $5 \pm 2$ & $19 \pm 4$ & $23 \pm 5$ \\
\hline $0736+053$ & Procyon B & $\mathrm{DA}+\mathrm{F} 5 \mathrm{IV}-\mathrm{V}$ & J0739.3+0513 & 200437 & 3.8 & good & $11700 \pm 110$ & $874 \pm 30$ & $118 \pm 11$ \\
\hline $0800-477 \mathrm{~J}$ & & DA & J0800.4-4745 & 400158 & 2.4 & good & $1333 \pm 37$ & $42 \pm 6$ & $0 \pm 0$ \\
\hline $0805+654$ & PG $0805+654$ & DA1 & J0809.6+6518 & 700258 & 8.4 & $\mathrm{U} 2$ & $198 \pm 14$ & $67 \pm 8$ & $18 \pm 4$ \\
\hline $0824+288$ & PG0824+289 & $\mathrm{DA}+\mathrm{dC}+\mathrm{M} 3$ & $\mathrm{~J} 0827.0+2844$ & 201083 & 2.7 & good & $586 \pm 24$ & $5 \pm 2$ & $1 \pm 1$ \\
\hline $0839-528$ & IC $2391 \mathrm{KR} 1$ & DA3 & J0841.1-5300 & 200501 & 22.9 & $\mathrm{U} 2,3$ & $16 \pm 4$ & $14 \pm 4$ & $30 \pm 6$ \\
\hline $0840+200$ & LB 1876 & DA5 & J0842.8+1951 & 200250 & 1.9 & $\mathrm{U} 2$ & $11 \pm 3$ & $4 \pm 2$ & $10 \pm 3$ \\
\hline $0841+033 \mathrm{~J}$ & RE J0841+032 & DA1 & J0841.0+0320 & 201362 & 0.3 & good & $182 \pm 13$ & $1 \pm 1$ & $0 \pm 0$ \\
\hline $0842+490$ & HD 74389B & $\mathrm{DA}+\mathrm{A} 2 \mathrm{~V}$ & J0845.8+4852 & 200816 & 0.3 & good & $91 \pm 10$ & $3 \pm 2$ & $0 \pm 0$ \\
\hline $0903+166$ & $\ldots$ & $\ldots$ & J0905.9+1624 & 700385 & 6.2 & $\mathrm{U} 2,3$ & $21 \pm 5$ & $21 \pm 5$ & $12 \pm 3$ \\
\hline $0904+511$ & PG $0904+511$ & DA1.5 & J0907.7+5058 & 800474 & 9.6 & good & $524 \pm 23$ & $25 \pm 5$ & $26 \pm 5$ \\
\hline 0916-197J & RE J0916-194 & $\mathrm{DA}$ & J0916.9-1946 & 400162 & 2.9 & good & $1494 \pm 39$ & $19 \pm 4$ & $0 \pm 0$ \\
\hline $0937+505$ & PG 0937+505 & DA1 & J0940.3+5021 & 200957 & 4.1 & good & $239 \pm 16$ & $0 \pm 0$ & $0 \pm 0$ \\
\hline $0954+697$ & PG 0954+697 & DA2.5 & J0958.4+6928 & 600101 & 21.4 & $\mathrm{U} 4$ & $174 \pm 13$ & $76 \pm 9$ & $38 \pm 6$ \\
\hline $1010+064$ & PG $1010+065$ & DA1 & $\mathrm{J} 1013.4+0612$ & 200540 & 6.8 & good & $204 \pm 14$ & $5 \pm 2$ & $2 \pm 1$ \\
\hline $1032+534 \mathrm{~J}$ & RE J1032+535 & DA1 & $\mathrm{J} 1032.1+5330$ & 900149 & 17.7 & good & $40710 \pm 202$ & $335 \pm 18$ & $81 \pm 9$ \\
\hline $1040+451$ & PG $1040+451$ & DA1 & $\mathrm{J} 1043.5+4454$ & 201020 & 14.7 & good & $1787 \pm 42$ & $66 \pm 8$ & $28 \pm 5$ \\
\hline $1059+514 \mathrm{~J}$ & & $\mathrm{DA}$ & $\mathrm{J} 1059.2+5124$ & 400159 & 3.2 & good & $6582 \pm 81$ & $102 \pm 10$ & $0 \pm 0$ \\
\hline $1109+244$ & PG $1109+244$ & DA1.5 & $\mathrm{J} 1112.6+2409$ & 201365 & 0.2 & good & $46 \pm 7$ & $0 \pm 0$ & $0 \pm 0$ \\
\hline $1134+300$ & GJ 433.1 & $\mathrm{DA} 2$ & $\mathrm{~J} 1137.0+2948$ & 200091 & 33.9 & good & $327 \pm 18$ & $59 \pm 8$ & $51 \pm 7$ \\
\hline $1144+004$ & $\ldots$ & DQZO1 & $\mathrm{J} 1146.5+0012$ & 201242 & 5.8 & good & $149 \pm 12$ & $5 \pm 2$ & $3 \pm 2$ \\
\hline
\end{tabular}


Table 1-Continued

\begin{tabular}{|c|c|c|c|c|c|c|c|c|c|}
\hline$(1)$ & $(2)$ & $(3)$ & $(4)$ & $\begin{array}{c}(5) \\
\text { ROSAT }\end{array}$ & $\begin{array}{c}(6) \\
\text { Exp. }\end{array}$ & $(7)$ & $\begin{array}{c}(8) \\
\text { X-ray Count }\end{array}$ & \multicolumn{2}{|c|}{ X-ray Counts Reported in WGACAT } \\
\hline WD & Common & WD & WGACAT & PSPC & Time & Pos. & $0.1-0.4 \mathrm{kev}$ & $0.4-0.9 \mathrm{kev}$ & $0.9-2.4 \mathrm{kev}$ \\
\hline Number & Name & Type & Number & Obs. \# & {$[\mathrm{ks}]$} & Coin. & {$[\mathrm{cts}]$} & {$[\mathrm{cts}]$} & [cts] \\
\hline $1159-034$ & GW Vir & DQZO.4 & J1201.7-0345 & 701202 & 13.6 & good & $779 \pm 28$ & $12 \pm 4$ & $13 \pm 4$ \\
\hline $1213+528$ & EG UMa & $\mathrm{DA} 4+\mathrm{dM} 2 \mathrm{e}$ & $\mathrm{J} 1215.6+5230$ & 200953 & 2.6 & good & $249 \pm 16$ & $68 \pm 8$ & $84 \pm 9$ \\
\hline $1229+290$ & $\ldots$ & $\mathrm{DC}$ & $\mathrm{J} 1231.7+2848$ & 201163 & 1.8 & $\mathrm{U} 1,3$ & $113 \pm 11$ & $44 \pm 7$ & $39 \pm 6$ \\
\hline $1234+481$ & PG $1234+481$ & DA1 & $\mathrm{J} 1236.7+4755$ & 200578 & 2.5 & good & $2127 \pm 46$ & $89 \pm 9$ & $0 \pm 0$ \\
\hline $1254+223$ & GD 153 & DA1 & $\mathrm{J} 1257.0+2201$ & 132471 & 8.6 & good & $16673 \pm 130$ & $95 \pm 10$ & $0 \pm 0$ \\
\hline $1255+258 \mathrm{~J}$ & HD 112313 & CSPN+G5III & $\mathrm{J} 1255.5+2553$ & 201514 & 18.8 & good & $90 \pm 9$ & $29 \pm 5$ & $39 \pm 6$ \\
\hline $1314+293$ & $\mathrm{HZ} 43 \mathrm{~A}$ & $\mathrm{DA} 1+\mathrm{dM} 3.5 \mathrm{e}$ & $\mathrm{J} 1316.3+2906$ & 100308 & 21.5 & good & $1585270 \pm 1260$ & $7312 \pm 86$ & $120 \pm 11$ \\
\hline $1317+453$ & GJ 2100 & DA3.5 & $\mathrm{J} 1319.2+4505$ & 900325 & 10.3 & $\mathrm{U} 1,2$ & $34 \pm 6$ & $12 \pm 4$ & $14 \pm 4$ ! \\
\hline $1325+581$ & EGGR 358 & DA7 & $\mathrm{J} 1327.6+5755$ & 600458 & 18.1 & $\mathrm{U} 2,4$ & $247 \pm 16$ & $35 \pm 6$ & $44 \pm 7 \stackrel{\omega}{\models}$ \\
\hline $1333+510$ & PG $1333+510$ & $\mathrm{DA}$ & $\mathrm{J} 1335.2+5049$ & 800047 & 16.4 & good & $447 \pm 21$ & $51 \pm 7$ & $47 \pm 7^{\prime}$ \\
\hline $1403-077$ & PG 1403-077 & DA1 & J1406.0-0758 & 200528 & 4.8 & good & $345 \pm 19$ & $9 \pm 3$ & $1 \pm 1$ \\
\hline $1446+634 \mathrm{~J}$ & $\ldots$ & DA1 & $\mathrm{J} 1446.0+6329$ & 700975 & 4.2 & good & $467 \pm 22$ & $5 \pm 2$ & $2 \pm 1$ \\
\hline $1501+664$ & RE J1502+661 & DZ1 & $\mathrm{J} 1502.1+6612$ & 170001 & 43.2 & good & $357416 \pm 600$ & $1689 \pm 41$ & $12 \pm 4$ \\
\hline $1522+662$ & $\ldots$ & $\mathrm{DO}$ & $\mathrm{J} 1522.9+6604$ & 201240 & 4.7 & good & $742 \pm 27$ & $13 \pm 4$ & $1 \pm 1$ \\
\hline $1620-391$ & EGGR 274 & DA2 & J1623.5-3913 & 200588 & 1.9 & good & $634 \pm 25$ & $2 \pm 1$ & $2 \pm 1$ \\
\hline $1631+781$ & 1ES $1631+78.1$ & $\mathrm{DA} 1+\mathrm{dM} 4 \mathrm{e}$ & $\mathrm{J} 1629.1+7804$ & 170154 & 37.5 & good & $125636 \pm 350$ & $1355 \pm 37$ & $350 \pm 19$ \\
\hline $1633+572$ & GJ 630.1B & $\mathrm{DQ} 8+\mathrm{dM} 4 \mathrm{e}$ & $\mathrm{J} 1634.3+5709$ & 200721 & 47.5 & good & $2397 \pm 49$ & $1249 \pm 35$ & $1038 \pm 32$ \\
\hline $1634-573$ & HD 149499B & $\mathrm{DOZ} 1+\mathrm{K} 2 \mathrm{Ve}$ & J1638.5-5728 & 200773 & 1.4 & good & $240 \pm 16$ & $145 \pm 12$ & $146 \pm 12$ \\
\hline $1636+351$ & PG 1636+351 & DA1.5 & $\mathrm{J} 1638.4+3500$ & 201082 & 4.2 & good & $909 \pm 30$ & $9 \pm 3$ & $0 \pm 0$ \\
\hline $1641+387$ & GD 357 & DA3 & $\mathrm{J} 1643.1+3840$ & 201538 & 5.5 & U1 & $26 \pm 5$ & $10 \pm 3$ & $13 \pm 4$ \\
\hline $1650+406 \mathrm{~J}$ & RE J1650+403 & DA1 & $\mathrm{J} 1650.3+4037$ & 201080 & 2.2 & good & $55 \pm 7$ & $1 \pm 1$ & $0 \pm 0$ \\
\hline $1657+343$ & $\mathrm{PG} 1657+343$ & DA2 & $\mathrm{J} 1658.8+3418$ & 201079 & 6.4 & good & $591 \pm 24$ & $7 \pm 3$ & $0 \pm 0$ \\
\hline $1658+440$ & PG $1658+440$ & DAp1 & $\mathrm{J} 1659.8+4400$ & 201078 & 6.7 & good & $503 \pm 22$ & $7 \pm 3$ & $1 \pm 1$ \\
\hline $1659+442$ & PG $1659+442$ & $\mathrm{DA}$ & $\mathrm{J} 1700.6+4410$ & 201078 & 6.7 & $\mathrm{U} 1,2$ & $22 \pm 5$ & $7 \pm 3$ & $7 \pm 3$ \\
\hline
\end{tabular}


Table 1-Continued

\begin{tabular}{|c|c|c|c|c|c|c|c|c|c|}
\hline (1) & $(2)$ & $(3)$ & (4) & $\begin{array}{c}(5) \\
\text { BOSAT }\end{array}$ & $(6)$ & (7) & (8) & (9) & $\begin{array}{c}(10) \\
(10 A C A T\end{array}$ \\
\hline WD & Common & WD & WGACAT & PSPC & Time & Pos. & $0.1-0.4 \mathrm{kev}$ & $0.4-0.9 \mathrm{kev}$ & $0.9-2.4 \mathrm{kev}$ \\
\hline Number & Name & Type & Number & Obs. \# & {$[\mathrm{ks}]$} & Coin. & {$[\mathrm{cts}]$} & {$[\mathrm{cts}]$} & {$[\mathrm{cts}]$} \\
\hline $1802+213$ & GD 372 & DA4 & $\mathrm{J} 1804.4+2120$ & 200940 & 13.7 & $\mathrm{U} 2,3$ & $35 \pm 6$ & $21 \pm 5$ & $14 \pm 4$ \\
\hline $1821+643$ & DS Dra & DOZ.4 & $\mathrm{J} 1821.8+6422$ & 700948 & 2.0 & good & $135 \pm 12$ & $9 \pm 3$ & $20 \pm 5$ \\
\hline $1844-654$ & $\ldots$ & DA & J1848.9-6525 & 200941 & 16.0 & good & $757 \pm 28$ & $126 \pm 11$ & $64 \pm 8$ \\
\hline $1906-600$ & $\ldots$ & $\mathrm{DC}$ & J1910.8-5958 & 300047 & 5.2 & U3 & $7 \pm 3$ & $20 \pm 5$ & $41 \pm 6$ \\
\hline $1910+047$ & $\ldots$ & DA2 & $\mathrm{J} 1912.5+0452$ & 400271 & 2.8 & $\mathrm{U} 4$ & $20 \pm 45$ & $10 \pm 3$ & $27 \pm 5$ \\
\hline $1944-421$ & V3885 Sgr & $\mathrm{CV}$ & J1947.6-4200 & 300232 & 10.4 & good & $345 \pm 19$ & $448 \pm 21$ & $699 \pm 26$ \\
\hline $1957+225$ & $\ldots$ & $\mathrm{DAO}$ & J1959.6+2243 & 900016 & 5.9 & good & $828 \pm 29$ & $5 \pm 2$ & $0 \pm 0$ \\
\hline $2013+400 \mathrm{~J}$ & RE J2013+400 & $\mathrm{DAO}$ & $\mathrm{J} 2013.1+4002$ & 400157 & 3.0 & good & $1166 \pm 34$ & $17 \pm 4$ & $9 \pm 3$ \\
\hline $2014-575$ & RE J2018-572 & DA2 & J2018.8-5721 & 200580 & 4.1 & good & $148 \pm 12$ & $3 \pm 2$ & $0 \pm 0 \quad \stackrel{0}{N}$ \\
\hline $2020-425$ & UVE J2024-42.4 & DA & J2023.9-4224 & 200488 & 0.9 & good & $71 \pm 8$ & $2 \pm 1$ & $0 \pm 0$ \\
\hline $2028+390$ & GD 391 & DA2 & J2029.9+3913 & 200412 & 2.2 & good & $46 \pm 7$ & $0 \pm 0$ & $0 \pm 0$ \\
\hline $2032+248$ & HD 340611 & DA2.5 & $\mathrm{J} 2034.3+2504$ & 200087 & 9.9 & good & $100 \pm 10$ & $2 \pm 1$ & $5 \pm 2$ \\
\hline $2034-275 \mathrm{~J}$ & & DA & J2034.9-2734 & 201236 & 5.0 & good & $574 \pm 24$ & $4 \pm 2$ & $2 \pm 1$ \\
\hline $2052+466 \mathrm{~J}$ & & $\mathrm{DO}$ & J2052.6+4639 & 200114 & 3.5 & U3 & $801 \pm 28$ & $118 \pm 11$ & $27 \pm 5$ \\
\hline $2056+033$ & PG 2056+033 & DA1 & J2058.7+0332 & 200955 & 5.6 & good & $37 \pm 6$ & $2 \pm 1$ & $1 \pm 1$ \\
\hline $2111+498$ & GD 394 & DA1.5 & $\mathrm{J} 2112.7+5006$ & 200427 & 0.4 & good & $207 \pm 14$ & $1 \pm 1$ & $0 \pm 0$ \\
\hline $2117+342 \mathrm{~J}$ & V2027 Cyg & DO & $\mathrm{J} 2117.1+3412$ & 201512 & 26.6 & good & $6588 \pm 81$ & $194 \pm 14$ & $66 \pm 8$ \\
\hline $2153-419$ & RE J2156-414 & DA & J2156.5-4142 & 200487 & 0.7 & good & $397 \pm 20$ & $2 \pm 1$ & $0 \pm 0$ \\
\hline $2154-512$ & GJ 841B & DQ7+dM3-5e & J2157.7-5059 & 600146 & 5.1 & good & $1851 \pm 43$ & $606 \pm 25$ & $706 \pm 27$ \\
\hline $2309+105$ & GD 246 & DA1 & $\mathrm{J} 2312.3+1047$ & 100578 & 10.3 & good & $36832 \pm 190$ & $141 \pm 13$ & $3 \pm 2$ \\
\hline $2321-549$ & RE J2324-544 & DA & J2324.5-5441 & 400166 & 4.1 & good & $1964 \pm 44$ & $20 \pm 5$ & $1 \pm 1$ \\
\hline $2357+296$ & PG 2357+296 & DA1 & J0000.1+2956 & 200535 & 3.8 & good & $36 \pm 6$ & $2 \pm 1$ & $1 \pm 1$ \\
\hline
\end{tabular}


Table 2. White Dwarfs with Hard X-ray Emission

\begin{tabular}{llccll}
\hline \hline $\begin{array}{l}\text { WD } \\
\text { Number }\end{array}$ & $\begin{array}{l}\text { Spectral } \\
\text { Type }\end{array}$ & $\begin{array}{l}\text { Parallax } \\
(\mathrm{mas})\end{array}$ & $\begin{array}{l}\log a^{\mathrm{b}} \\
(\mathrm{AU})\end{array}$ & $\begin{array}{l}k T^{\mathrm{c}} \\
(\mathrm{keV})\end{array}$ & $\begin{array}{l}\log L_{\mathrm{X}}^{\mathrm{d}} \\
\left(\mathrm{ergs} \mathrm{s}^{-1}\right)\end{array}$ \\
\hline $0216-032$ & DA+M2-7III & $7.79 \pm 1.07$ & $2.04:$ & 0.5 & 29.9 \\
$0339-451$ & DA & $\ldots$ & $\ldots$ & $\ldots$ & $\ldots$ \\
$0347+171$ & DA2+K2V & $21.37 \pm 1.62$ & -1.81 & 0.8 & 29.8 \\
$0429+176$ & DA2+dM4.5e & $21.58^{\mathrm{e}}$ & -1.91 & 0.6 & 28.6 \\
$0736+053$ & DA+F5IV-V & $285.93 \pm 0.88$ & 1.20 & 0.2 & 27.3 \\
$1134+300$ & DA2+(AGN) & $65.28 \pm 3.61$ & $\ldots$ & $\ldots$ & $\ldots$ \\
$1159-034$ & DQZO.4 & $\ldots$ & $\ldots$ & $\ldots$ & $\ldots$ \\
$1213+528$ & DA4+dM2e & $33.4 \pm 4.1^{\mathrm{f}}$ & -1.89 & 0.8 & 28.8 \\
$1234+481$ & DA1 & $6.3^{\mathrm{g}}$ & $\ldots$ & $\ldots$ & $\ldots$ \\
$1255+258 \mathrm{~J}$ & CSPN+G5III & $4.70 \pm 0.75^{\mathrm{h}}$ & $>0$. & 0.7 & 29.3 \\
$1314+293$ & DA1+dM3.5e & $31.26 \pm 8.33$ & $\geq 1.85$ & 0.6 & 28.0 \\
$1333+510$ & DA & $\ldots$ & $\ldots$ & $\ldots$ & $\ldots$ \\
$1631+781$ & DA1+dM4e & $19 . \mathrm{g}^{\mathrm{g}}$ & $>0$. & 0.8 & 28.7 \\
$1633+572$ & DQ8+2(dM3-4e) & $68.4 \pm 3.3^{\mathrm{i}}$ & $\geq 2.58$ & 0.4 & 28.0 \\
$1634-573$ & DOZ1+K2Ve & $26.94 \pm 1.88^{\mathrm{j}}$ & $\geq 1.69$ & 0.7 & 29.3 \\
$1944-421$ & CV & $9.11 \pm 1.95$ & -2.09 & 1.2 & 30.3 \\
$2154-512$ & DQ7+2(dM3-5e) & $61.63 \pm 2.67^{\mathrm{k}}$ & $\geq 2.66$ & 0.8 & 29.0 \\
\hline
\end{tabular}

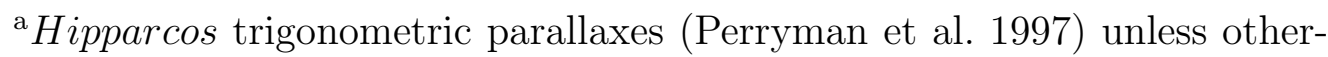
wise noted.

${ }^{\mathrm{b}}$ These values are derived from visual orbits, apparent separations, or orbital periods cited in $\S 3.1$, supplemented by parallaxes tabulated here and, for short-period systems, mass estimates from Ritter \& Kolb (1998). For V471 Tau $=$ WD 0347+171, masses were adopted from O’Brien, Bond, \& Sion (2001).

${ }^{\mathrm{c}}$ For $T=10^{6} \mathrm{~K}, k T=0.086 \mathrm{keV}$.

${ }^{\mathrm{d}}$ In the $0.5-2.4 \mathrm{keV}$ energy band.

eAssumes membership in the Hyades (van Altena 1969) with distance from Perryman et al. (1998).

${ }^{\mathrm{f}}$ Trigonometric parallax from Dahn et al. (1982).

gParallax deduced from EUV/IR photometric models of Green, Ali, \& Napiwotzki (2000).

h Trigonometric parallax from Harris et al. (1997). 
Table 3. Multi-band Photometry of White Dwarfs with Hard X-rays

\begin{tabular}{lllllll}
\hline \hline $\begin{array}{l}\text { WD } \\
\text { Number }\end{array}$ & $\begin{array}{l}\text { Spectral } \\
\text { Type }\end{array}$ & $\begin{array}{l}V \\
(\mathrm{mag})\end{array}$ & $\begin{array}{l}J \\
(\mathrm{mag})\end{array}$ & $\begin{array}{l}H \\
(\mathrm{mag})\end{array}$ & $\begin{array}{l}K \\
(\mathrm{mag})\end{array}$ & $\begin{array}{l}\text { 2MASS }^{\mathrm{a}} \\
\text { Source }\end{array}$ \\
\hline $0005+511$ & DO & 13.32 & 13.93 & 14.13 & 14.18 & $\mathrm{~J} 0008181+512316$ \\
$0216-032$ & DA+M2-7III & $11.32^{\mathrm{b}}$ & -1.06 & -1.89 & -2.44 & $\mathrm{~J}_{2192081-025841^{\mathrm{c}}}$ \\
$0429+176$ & DA2+dM4.5e & 13.93 & 10.76 & 10.12 & 9.93 & $\mathrm{~J}^{\mathrm{d}} 0432237+174502$ \\
$1134+300$ & DA2+(AGN) & 12.50 & 12.95 & 13.04 & 13.13 & $\mathrm{~d}$ \\
$1159-034$ & DQZO.4 & $14.87^{\mathrm{e}}$ & 15.58 & 15.87 & 15.78 & $\mathrm{~J} 1201459-034540$ \\
$1234+481$ & DA1 & 14.42 & 14.99 & 15.07 & 15.12 & $\mathrm{~J} 1236451+475522$ \\
$1631+781$ & DA1+dM4e & $13^{\mathrm{f}}$ & 11.00 & 10.28 & 10.15 & $\mathrm{~J} 1629102+780439$ \\
$1633+572$ & DQ8+ & 14.99 & 14.09 & 14.08 & 14.07 & $\mathrm{~J} 1634216+571008$ \\
& 2(dM3-4e) & $12.87^{\mathrm{g}}$ & 8.50 & 8.04 & 7.77 & $\mathrm{~J} 1634204+570943$ \\
$2226-210$ & DAO & 13.54 & 14.35 & 14.50 & 14.62 & $\mathrm{~J} 2229385-205013$ \\
\hline
\end{tabular}

${ }^{a}$ The Two Micron All Sky Survey (2MASS) is a joint project of the University of Massachusetts and the Infrared Processing and Analysis Center/California Institute of Technology.

${ }^{\mathrm{b}}$ Hubble Space Telescope $F 550 M$ magnitude, equivalent to Strömgren $y$ magnitude, of the white dwarf alone (Karovska et al. 1997).

${ }^{c}$ Saturated in 2MASS survey. $J, H$, and $K$ from Catchpole et al. (1979). Variable in all bandpasses.

${ }^{\mathrm{d}} V$ - Massey \& Gronwall (1990); $J, H$, and $K$ - Hawarden et al. (2001).

${ }^{\mathrm{e}} y$ magnitude.

${ }^{\mathrm{f}} B$ magnitude.

${ }^{\mathrm{g}}$ Out-of-eclipse maximum. 
Table 4. Properties of the Hottest Apparently Single White Dwarfs

\begin{tabular}{lllllr}
\hline \hline WD Number & Spectral Type $^{\mathrm{a}}$ & \multicolumn{1}{c}{$\begin{array}{c}T_{\text {eff }}{ }^{\mathrm{b}} \\
{[\mathrm{K}]}\end{array}$} & $\begin{array}{c}V^{\mathrm{a}} \\
{[\mathrm{Mag}]}\end{array}$ & X-Ray $^{\mathrm{c}}$ & $\begin{array}{c}\text { Exp. Time } \\
{[\mathrm{ks}]}\end{array}$ \\
\hline WD 0005+511 & DO & 120,000 & 13.32 & $\mathrm{~S}, \mathrm{H}$ & 5.0 \\
WD 0044-121 & PG 1159 & 150,000 & 11.84 & $\mathrm{~S}$ & 11.4 \\
WD 0122-753J & DO & 180,000 & 15.4 & $\mathrm{~S}, \mathrm{H} ?$ & 5.6 \\
WD 0726+133 & PG 1159 & $140,000^{\mathrm{e}}$ & 15.99 & $\mathrm{ND}$ & 2.8 \\
WD 1144+004 & DQZO1 & 150,000 & 15.10 & $\mathrm{~S}$ & 5.8 \\
WD 1159-034 & DQZO.4 & 140,000 & 14.84 & $\mathrm{~S}, \mathrm{H}$ & 13.6 \\
WD 1501+664 & DZ1 & 170,000 & 15.9 & $\mathrm{~S}$ & 43.2 \\
WD 1522+662 & DO & 140,000 & $16.4^{\mathrm{f}}$ & $\mathrm{S}$ & 4.7 \\
WD 1821+643 & DOZ.4 & $140,000^{\mathrm{g}}$ & 15.04 & $\mathrm{~S}$ & 3.1 \\
WD 1957+225 & DAO & 108,600 & 14.2 & $\mathrm{~S}$ & 19.9 \\
WD 2117+342J & DO & 170,000 & 13.16 & $\mathrm{~S}$ & 26.6 \\
WD 2226-210 & DAO & 103,600 & 13.4 & $\mathrm{~S}, \mathrm{H}$ & 4.9 \\
WD 2333+301 & DOZ.3 & $150,000^{\mathrm{e}}$ & 16.13 & $\mathrm{ND}$ & 4.2 \\
\hline
\end{tabular}

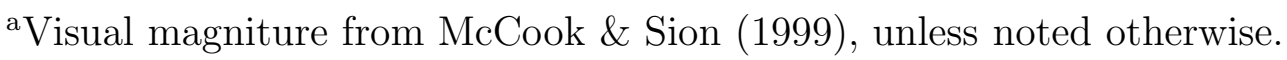

bStellar effective temperature from Napiwotzki (1999), unless noted otherwise.

'X-ray emission detected by ROSAT PSPC observations. ND: not detected;

$\mathrm{S}$ : detected in the $0.1-0.5 \mathrm{keV}$ band; $\mathrm{H}$ : detected in the $0.6-2.4 \mathrm{keV}$ band.

${ }^{\mathrm{d}}$ Exposure time of available ROSAT PSPC observation.

${ }^{\text {e}}$ From Werner et al. (1997).

${ }^{\mathrm{f}} B$ magnitude from McCook \& Sion (1999).

${ }^{\text {g} F r o m ~ K o e s t e r k e, ~ D r e i z l e r, ~ \& ~ R a u c h ~(1998) . ~}$ 

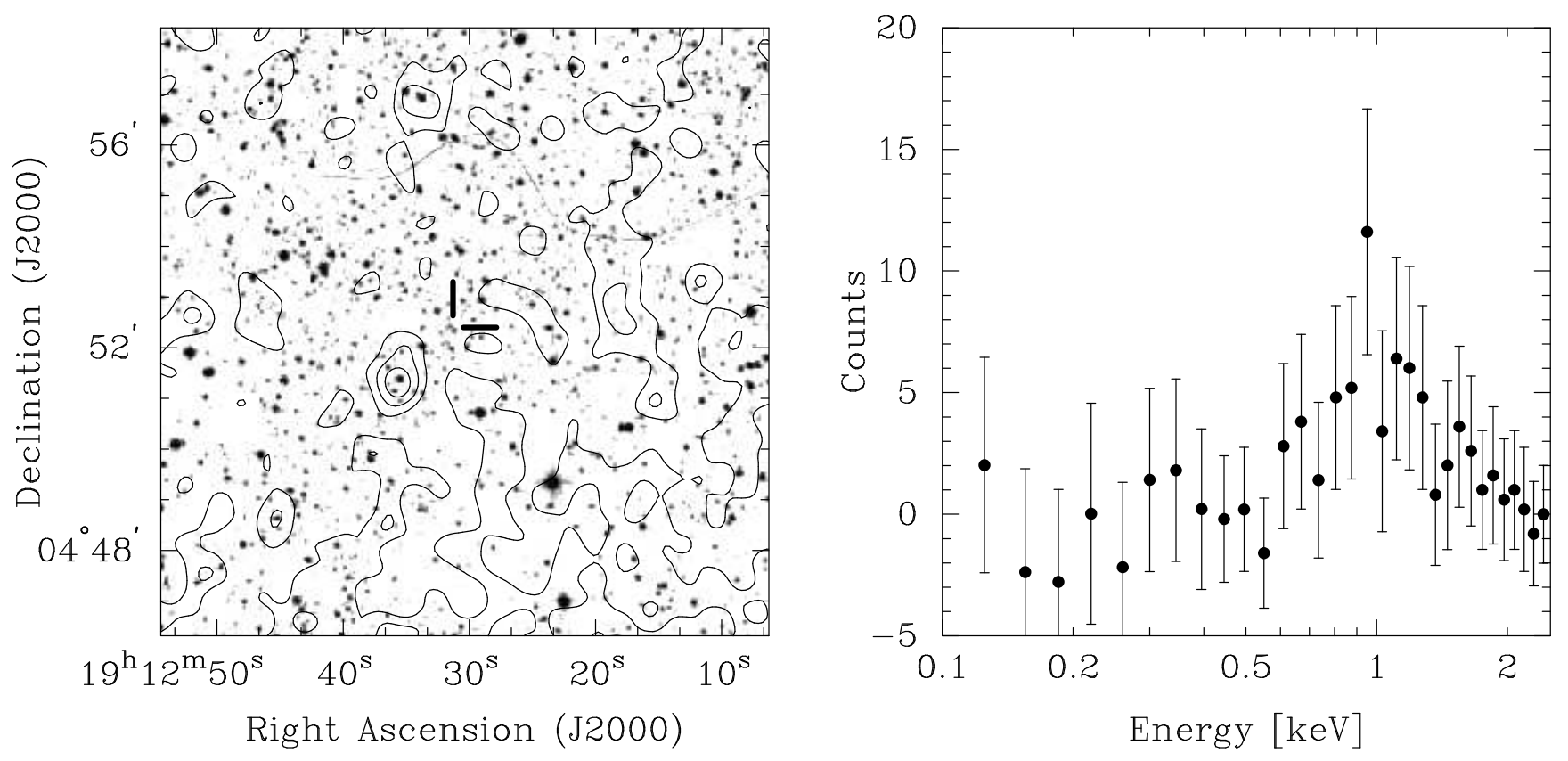

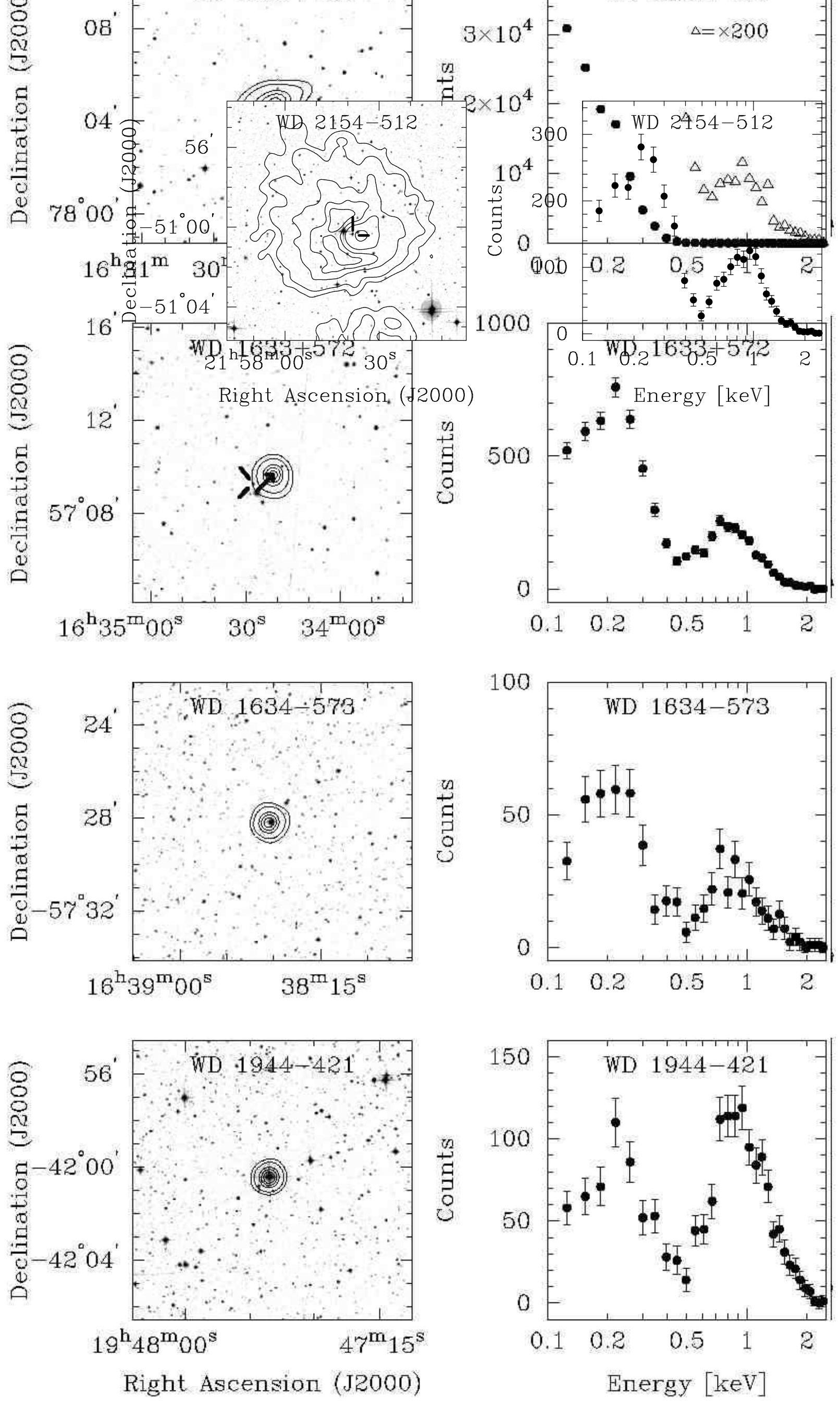

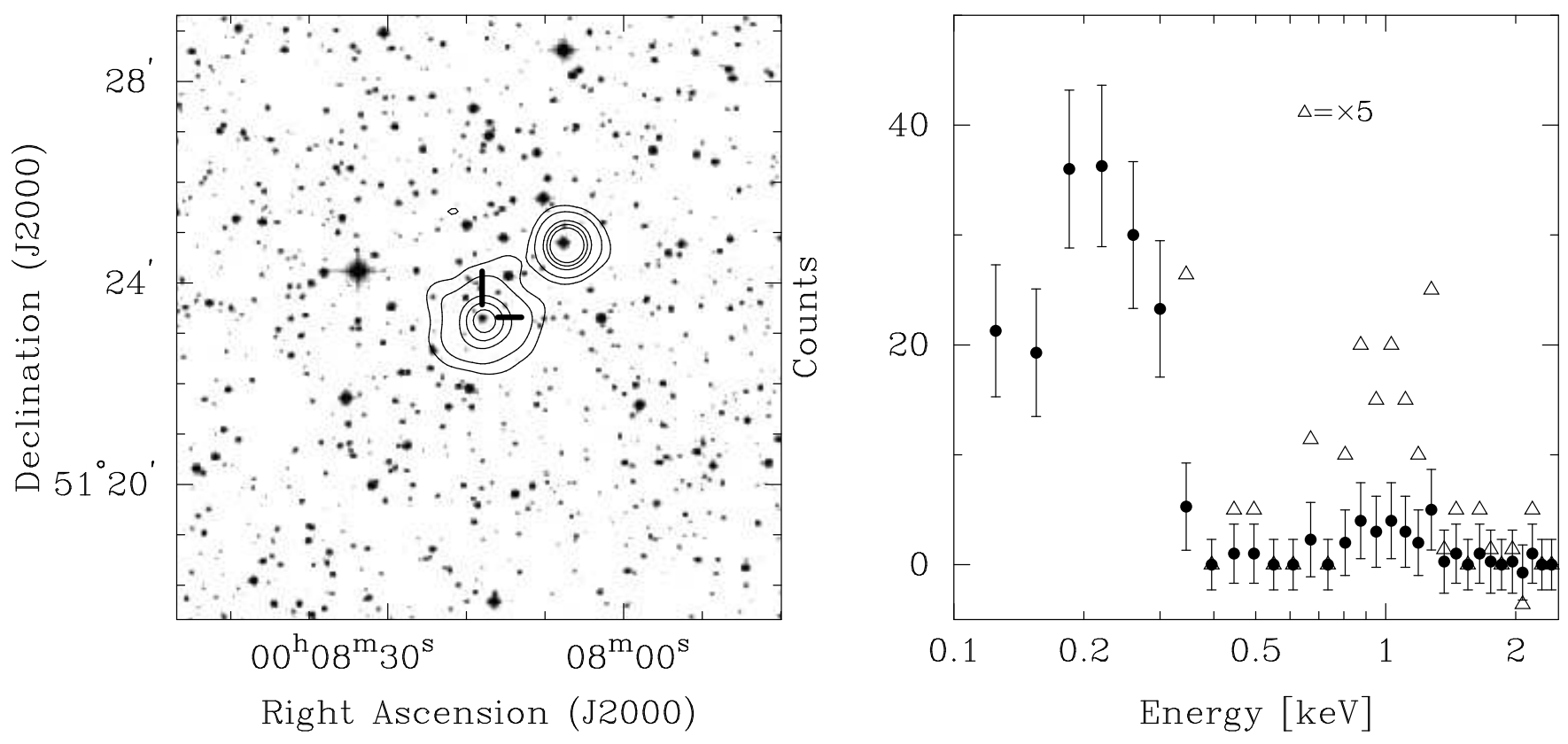

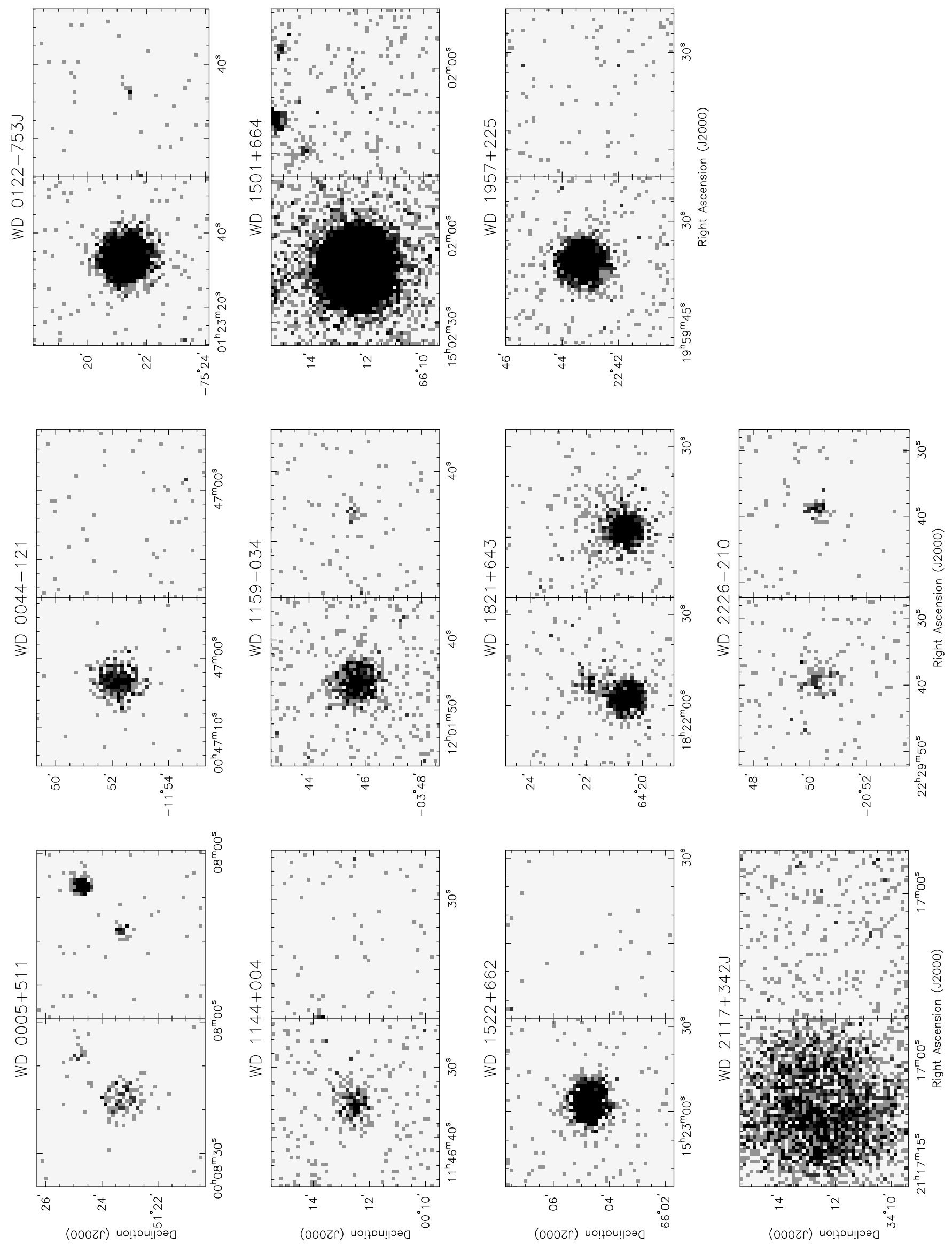